\section{Prevalência de doação de sangue e fatores associados em Florianópolis, Sul do Brasil: estudo de base populacional}

\author{
Prevalence of blood donation and associated \\ factors in Florianópolis, Southern Brazil: a \\ population-based study
}

\section{Prevalencia de la donación de sangre y factores asociados en Florianópolis, sur de Brasil: un estudio basado en la población}

Rafael Mariano Gislon da Silva ${ }^{1}$

Emil Kupek ${ }^{1}$

Karen Glazer Peres 1

\footnotetext{
1 Universidade Federal de Santa Catarina,

Florianópolis, Brasil.

Correspondência R. M. G. Silva Universidade Federal de Santa Catarina. Rua Coronel Lica Ramos 258 Lages, SC 88508-320, Brasil. rafaelmarianogislon@ hotmail.com
}

\begin{abstract}
The main objectives of this study were to estimate the prevalence of blood donation and to identify associated factors. A populated-based cross-sectional study was carried out $(n=1,720)$ including subjects 20 to 59 years of age in Florianópolis, Santa Catarina State, Brazil. Blood donation any time in life and in the previous year was reported by $30.6 \%$ and $6.2 \%$ of respondents, respectively. Among the latter, 31.8\% reported repeat donation (at least twice in the previous year), $80.4 \%$ reported voluntary donation, and 15.9\% replacement donation. Multivariate Poisson regression $(p<0.05)$ showed that male gender, black or brown self-reported skin color, higher age group, higher educational level, and living with other blood donors were all associated with ever donating blood, whereas younger age, single marital status, and higher educational level were associated with donating in the previous year. We found higher prevalence of blood donation in the previous year and higher percentage of young donors in this group, as well as lower percentage of replacement donors than previously reported in Brazil.
\end{abstract}

Blood Donors; Adult; Prevalence;

Cross-Sectional Studies

\section{Resumo}

Os principais objetivos foram estimar a prevalência de doação de sangue e identificar fatores associados. Foi realizado estudo de base populacional ( $n=1.720$ ), incluindo participantes com idade de 20 a 59 anos, residentes em Florianópolis, Região Sul do Brasil. Os participantes relataram doação de sangue alguma vez em 30,6\% dos casos, e nos últimos 12 meses em 6,2\% dos casos. Entre os últimos, 31,8\% afirmaram doação de repetição (mais de uma vez ao ano), 80,4\% doação espontânea e 15,9\% doação para reposição. De acordo com a regressão de Poisson $(p<0,05)$, sexo masculino, cor da pele autorreferida preta ou parda, maior faixa etária, maior escolaridade e coabitação com doador de sangue estiveram associados à doação alguma vez. Menor faixa etária e estado conjugal solteiro e maior escolaridade estiveram associados com doação nos últimos 12 meses. Constatamos maior prevalência de doação e maior percentagem de doadores mais jovens nesse grupo, assim como menor porcentagem de doadores de reposição do que previamente descrito no Brasil.

Doadores de Sangue; Adulto; Prevalência; Estudos Tranversais 


\section{Introdução}

O uso de sangue e hemoderivados desempenha importante papel na prática médica contemporânea. Países e regiões onde são realizados maior número de procedimentos médicos avançados, como cirurgia cardíaca, transplantes e quimioterapia, apresentam maior demanda por sangue 1 . Em 2001, aproximadamente 4,9 milhões de pacientes receberam unidades de sangue total ou concentrado de hemácias nos Estados Unidos 2. Naquele ano, 12,7\% dos hospitais americanos reportaram cancelamento de cirurgias eletivas por falta de hemocomponentes 2 . Regiões onde a hemoterapia é utilizada especialmente para o tratamento de complicações obstétricas, anemia e tratamento de traumatismos, necessitam menos transfusões 1. A taxa de doação (doações pela população acima de 18 anos) também tem sido menor em países com menor desenvolvimento tecnológico ${ }^{1}$. Esses países devem promover o aumento do número de doadores, para acompanhar seu desenvolvimento e consequente demanda por sangue. Não existem dados disponíveis sobre a demanda por sangue no Brasil, porém se estima que sejam realizadas cerca de 3 milhões e seiscentas mil transfusões todos os anos ${ }^{3}$. Acredita-se que estados com maior desenvolvimento tecnológico, como Rio de Janeiro, São Paulo e Rio Grande do Sul apresentem maior demanda por sangue 4 . A cidade de Florianópolis, além de importante centro urbano de Santa Catarina, conta com infraestrutura hospitalar de alta complexidade, sendo realizadas cirurgias cardíacas, quimioterapia e transplante de órgãos. Dessa forma, também supomos maior demanda por sangue na região.

Os benefícios da hemoterapia devem ser considerados em paralelo aos seus riscos, tais como a transmissão de doenças infecciosas. Políticas específicas de recrutamento, triagem clínica rigorosa e exames laboratoriais com alta sensibilidade podem diminuir este risco 1 . Estudo recente, em hemocentros de São Paulo, Minas Gerais e Pernambuco demonstrou risco residual para transmissão de HIV de 1,1 para 100 mil transfusões 5. Outro estudo, conduzido em Santa Catarina, demonstrou risco para transmissão de hepatite B por transfusão de 3,2 para cada 100 mil transfusões ${ }^{6}$. Tais riscos são maiores do que aqueles observados em países desenvolvidos 5,6. Supõe-se que essa diferença ocorra por conta da maior prevalência de indivíduos infectados na população geral, maior frequência de indivíduos que doam sangue pela primeira vez (primo doadores), maior frequência de doações para reposição ou pela expectativa de receberem resultados de exames sobre seu estado de saúde. Entretanto, os estudos publicados sobre o perfil do doador brasileiro tem sido esporádicos. Busca nas bases de dados MEDLINE e PubMed evidenciou apenas um estudo com base populacional nacional, na cidade de Pelotas, no Rio Grande do Sul 7. Os objetivos do nosso estudo foram determinar a prevalência de doação de sangue, o perfil dos participantes que referiram doação nos últimos 12 meses e os fatores associados à doação entre adultos residentes na área urbana de Florianópolis, Santa Catarina.

\section{Método}

Foi realizado estudo transversal, de base populacional, na área urbana do Município de Florianópolis, Região Sul do Brasil, no ano de 2009. O estudo foi parte de um projeto maior, destinado a avaliar a prevalência de agravos e fatores de risco à saúde da população adulta - Estudo EpiFloripa Adulto.

A população de referência foi composta por todos os adultos de 20-59 anos de idade residentes em Florianópolis. Essa população foi estimada em 249.530 pessoas, de acordo com o último censo populacional (Instituto Brasileiro de Geografia e Estatística. http://www.ibge.gov.br, acessado em 01/Jul/2009). Os parâmetros para cálculo do tamanho da amostra foram: prevalência estimada máxima (50\%), erro amostral de 4,0 pontos percentuais e nível de $95 \%$ de confiança. Adicionalmente, foi utilizado ajuste por efeito de delineamento (deff) igual a 2, adicionados $10 \%$ por causa de eventuais perdas ou recusas, $20 \%$ para controle de fatores de confusão e aumento do poder da amostra, elevando o total da amostra calculada para 2.016 participantes. O processo de amostragem foi realizado em conglomerados, em dois estágios. As unidades do primeiro estágio foram os setores censitários de recenseamento do Instituto Brasileiro de Geografia e Estatística (IBGE), constituídas por aproximadamente 300 domicílios cada uma. Os 420 setores urbanos de Florianópolis, de acordo com o Censo Demográfico de 2000 (http:/ / www.ibge.gov.br, acessado em 02/Mai/2010), foram estratificados em ordem crescente de renda, e foram sorteados sistematicamente 60 deles (com uma fração de amostragem igual a sete, selecionaram-se seis setores em cada decil em renda). As unidades do segundo estágio foram os domicílios. Uma etapa de arrolamento dos setores foi necessária para atualização dos domicílios, em razão do tempo decorrido desde o censo anterior à pesquisa. $\mathrm{Pa}$ ra tanto, procedeu-se à contagem de todos os domicílios habitados em cada um dos setores censitários sorteados. Para definir a unidade amos- 
tral, os domicílios foram sorteados com uma fração de amostragem igual a oito, totalizando 2.094 domicílios sorteados. Nos domicílios sorteados, foram entrevistados os habitantes dentro da faixa etária do estudo, sem problemas físicos ou mentais que impossibilitassem responder ao questionário, residentes a maior parte do tempo no domicílio e que concordassem em participar. Foram realizadas 1.720 entrevistas $(85,3 \%$ do previsto). Entretanto, a distribuição por decis de renda familiar, sexo e faixa etária da amostra foi semelhante àquela registrada para a população de Florianópolis pelo Censo Demográfico em 2010 (http://www.ibge.gov.br, acessado em 02/Mai/2010). Essa semelhança sugere que não ocorreu viés de seleção significativo devido às perdas ou recusas.

Os entrevistadores eram do sexo feminino, com ensino médio completo. $\mathrm{O}$ instrumento de coleta de dados foi um questionário padronizado, aplicado pelo entrevistador, que visava estimar a prevalência de agravos e fatores de risco à saúde, composto por 234 questões, incluindo questões sobre doação de sangue. Após as entrevistas, $15 \%$ dos participantes foram novamente entrevistados pelo supervisor e responderam a 10 perguntas estáveis, para averiguar a confiabilidade dos dados colhidos pelo primeiro entrevistador. A concordância entre as respostas obtidas pelos entrevistadores e pelo supervisor variou de substancial à excelente (kappa 0,6 a $0,9)$ 8. As questões relativas à doação de sangue eram: (1) haver doado sangue alguma vez; (2) algum dos moradores do domicílio haver doado sangue alguma vez; (3) questões sobre motivação da doação (doação espontânea, de reposição, pela expectativa de receber resultados de exames sobre seu estado de saúde, ou outros motivos); (4) em caso de doação, há quanto tempo doou pela última vez; e (5) quantas vezes doou no último ano. Foi considerada motivação de doação para reposição quando o participante referiu doação motivada pelo uso imediato de sangue por alguém relevante. A doação de sangue realizada com frequência maior ou igual a duas vezes nos últimos 12 meses foi considerada doação de repetição.

A prevalência de doação alguma vez na vida foi estimada pelo quociente entre o total de participantes que manifestaram doação de sangue alguma vez na vida pelo total de entrevistados que manifestaram haver doado sangue alguma vez na vida, acrescido daqueles que manifestaram não haver doado sangue. A prevalência de doadores ativos foi estimada pelo quociente entre o total de participantes que manifestaram doação de sangue no último ano pelo total de entrevistados que manifestaram haver doado sangue no último ano, acrescido daqueles que negaram haver doado sangue no último ano.

Para análise das características associadas à doação, as variáveis dependentes foram: doação alguma vez na vida e doação de sangue nos últimos 12 meses. As variáveis independentes foram: sexo (masculino ou feminino), cor da pele autorreferida (branca, parda ou preta), faixa etária em anos completos (20-29 anos, 30-39 anos, 4049 anos, ou 50-59 anos), estado marital (casado ou união estável, solteiro, separado, divorciado, ou viúvo), anos completos de estudo (menor ou igual a 4 anos, entre 5 e 8 anos, entre 9 e 11 anos, ou a partir de 12 anos), renda familiar mensal per capita do domicílio (abaixo de $\mathrm{R} \$ 510,00$, entre $\mathrm{R} \$$ 510,00 e $\mathrm{R} \$ 1.499,00$, ou a partir de $\mathrm{R} \$ 1.500,00$ ) e condição de saúde autorreferida (muito boa ou boa, ou regular, ruim ou muito ruim). As categorias para cor ou raça foram adaptadas daquelas utilizadas pelo IBGE para o Censo Demográfico de 2000 (http://www.ibge.gov.br, acessado em 20/Nov/2012). As categorias cor amarela e raça indígena não foram incluídas na análise pelo pequeno número. Apenas 17 participantes referiram cor da pele amarela e 20 participantes referiram raça indígena $(2,1 \%$ do total). A medida de associação foi razão de prevalências, com respectivo intervalo de 95\% de confiança (IC95\%), estimada por modelo de regressão de Poisson. O nível de significância foi valor de $p<0,05$. A análise dos dados foi realizada no programa Stata, versão 9.0 (Stata Corp., College Station, Estados Unidos). $\mathrm{O}$ efeito de delineamento assim como os pesos individuais na análise foi incorporado para todas as estimativas obtidas. Para tanto, foi utilizado o comando survey do Stata. Para realização da análise ajustada, todas as variáveis independentes foram incluídas no modelo, independentemente da medida de associação observada na análise bruta. Após a coleta dos dados, foi verificado o poder da amostra das variáveis independentes, no aplicativo Statcalc do programa Epi Info, versão 7.0.34 (Centers for Disease Control and Prevention, Atlanta, Estados Unidos). Variáveis com poder amostral menor que $80 \%$ foram assinaladas nas tabelas de resultados.

\section{Resultados}

Quanto às características da amostra, 44,2\% dos participantes eram do sexo masculino; a média etária foi 38,1 anos, com desvio-padrão (DP) 11,61 anos; $86 \%$ referiram cor da pele branca; $60,6 \%$ eram casados ou apresentavam união estável, $29,2 \%$ solteiros e $10,1 \%$ separados, divorciados ou viúvos. A média de anos de estudo formal foi 13,4 anos (DP 41,5 anos); 30,2\% apresen- 
tavam renda per capita familiar mensal abaixo de $\mathrm{R} \$ 510,00,38,9 \%$ entre R\$ 510,00, e R \$ 1.499,00 e $30,9 \%$ acima de $\mathrm{R} \$ 1.500,00 ; 79,8 \%$ avaliavam sua saúde como boa ou muito boa; $20,2 \%$ avaliaram-na como regular, ruim ou muito ruim. Aproximadamente um terço referiu que outro morador da casa já havia doado sangue.

A prevalência de doação alguma vez na vida foi 30,6\% (IC95\%: 28,4; 32,8\%), e doação nos últimos 12 meses 6,2\% (IC95\%: 5,1; 7,4\%). Entre os participantes que referiram doação nos últimos 12 meses 80,4\% (IC95\%: 72,7; 88,0\%) declararam doação espontânea; 15,9\% (IC95\%: 8,8; 22,9\%) doação de sangue para reposição; e 31,8\% (IC95\%: 22,8; 40,7\%) doação de repetição. Nenhum deles declarou doação pela expectativa de receber resultados de exames sobre seu estado de saúde. A prevalência de doação alguma vez na vida foi $58 \%$ menor para o sexo feminino, e $26 \%$ maior entre participantes que referiram cor da pele preta e parda. Comparado aos participantes com faixa etária entre 20 a 29 anos, a prevalência de doação alguma vez na vida foi $54 \%$ maior entre 30 a 39 anos, $46 \%$ maior entre 40 a 49 anos e $48 \%$ maior entre 50 a 59 anos. Comparado aos participantes com 12 ou mais anos completos de estudo, a prevalência de doação alguma vez na vida foi $30 \%$ menor entre participantes com 5 a 8 anos completos de estudo, e $49 \%$ menor entre participantes com até 4 anos completos de estudo. A prevalência de doação alguma vez na vida foi $35 \%$ maior para aqueles que referiram residência com outro morador doador de sangue (Tabela 1). Participantes com 50 a 59 anos apresentaram $68 \%$ menor prevalência de doação nos últimos 12 meses do que aqueles com 20 a 29 anos de idade. Participantes com estado conjugal solteiro apresentaram prevalência de doação nos últimos 12 meses $65 \%$ maior do que os casados ou com união estável. Comparado aos participantes com 12 ou mais anos completos de estudo, a prevalência de doação nos últimos 12 meses foi $66 \%$ menor entre participantes com 5 a 8 anos completos, e $100 \%$ menor entre participantes com até 4 anos completos (Tabela 2). A Figura 1 destaca a distribuição percentual dos participantes que referiram doação nos últimos 12 meses conforme a faixa etária.

\section{Discussão}

A maior prevalência de doação alguma vez na vida em comparação com a prevalência de doação nos últimos 12 meses já era esperada, uma vez que a doação nos últimos 12 meses constitui uma fração da doação alguma vez na vida. A prevalência de doação alguma vez na vida observada em nosso estudo foi semelhante àquela descrita por outros autores, como Javadzadeh Shahshahani em Yazd, no Irã 9 e Zaller em Urumque, na China 10. O único estudo com base populacional no Brasil sobre doação de sangue, conduzido em Pelotas, Rio Grande do Sul, também obteve resultado semelhante quanto à prevalência de doação alguma vez na vida 7 . A diferença entre as prevalências de doação alguma vez na vida e doação nos últimos 12 meses reforça a necessidade de incentivo à manutenção da atividade de doação.

Quanto ao perfil dos doadores, associação entre o sexo masculino, escolaridade e doação de sangue alguma vez na vida já havia sido descrita em Pelotas 7 e nos Estados Unidos 11,12,13. Estudo com base populacional conduzido naquele país entre 2007 e 2008 identificou que os principais fatores de exclusão para doação de sangue entre as mulheres eram a presença de anemia, neoplasias, tatuagens e baixo peso para doação 14 . Os motivos para menor doação prévia entre as mulheres em Florianópolis são desconhecidos, mas é possível que aqueles fatores de exclusão estejam envolvidos. Não encontramos estudos prévios avaliando a relação entre escolaridade e motivação para doação de sangue. Contudo, os autores acreditam que o nível de escolaridade facilite a compreensão sobre a importância da doação de sangue. A associação entre maior faixa etária e doação prévia já era esperada. As oportunidades para haver doado sangue aumentam quanto maior a faixa etária.

Estudos realizados nos Estados Unidos sugerem que, naquele país, minorias apresentam menor prevalência de doação de sangue do que a população geral 14,15 . Os possíveis fatores associados são a falta de confiança nos serviços de coleta e menor compreensão do significado social da doação de sangue 15. Em Florianópolis, apesar de a maioria dos indivíduos autorreferirem cor da pele branca, pretos e pardos apresentaram maior prevalência de doação alguma vez na vida. Esse resultado reforça a existência de diferenças na questão racial brasileira e norte-americana.

Uma teoria que pode explicar porque a coabitação com outro doador de sangue esteve relacionada com o ato de doar é a Teoria do Comportamento Deliberado (Plenned Behavior Theory) 16. Essa teoria sugere que o comportamento é influenciado pelo contexto, na medida em que ele estabelece referências para o que é normal ou típico. Em nosso estudo, o contexto pode ter sido representado por indivíduo próximo, tido como importante para o sujeito que manifesta comportamento semelhante.

Dados da Organização Mundial da Saúde (OMS) indicam que na maior parte dos países desenvolvidos a taxa de doadores de sangue excede 
Tabela 1

Fatores associados à doação de sangue alguma vez na vida. Município de Florianópolis, Santa Catarina, Brasil, 2009.

\begin{tabular}{|c|c|c|c|c|c|c|}
\hline \multirow[t]{2}{*}{ Variável } & \multirow[t]{2}{*}{$\mathrm{n}$} & \multirow[t]{2}{*}{ Prevalência (\%) } & \multicolumn{2}{|c|}{ Análise bruta * } & \multicolumn{2}{|c|}{ Análise ajustada * } \\
\hline & & & $\mathrm{RP} * \star(I C 95 \%)$ & Valor de $p$ & $\mathrm{RP} \star \star ~(I C 95 \%)$ & Valor de $p$ \\
\hline \multicolumn{7}{|l|}{ Sexo } \\
\hline Masculino & 760 & 44,74 & 1,00 & & 1,00 & \\
\hline Feminino & 957 & 19,44 & $0,43(0,37 ; 0,51)$ & $<0,001$ & $0,42(0,35 ; 0,49)$ & $<0,001$ \\
\hline \multicolumn{7}{|l|}{ Cor da pele autorreferida } \\
\hline Branca & 1.441 & 30,26 & 1,00 & & 1,00 & \\
\hline Parda ou preta *** & 234 & 32,91 & $1,09(0,90 ; 1,31)$ & 0,377 & $1,26(1,06 ; 1,51)$ & 0,011 \\
\hline \multicolumn{7}{|l|}{ Idade (anos) } \\
\hline $20-29$ & 540 & 24,26 & 1,00 & & 1,00 & \\
\hline $30-39$ & 391 & 35,81 & $1,46(1,21 ; 1,80)$ & $<0,001$ & $1,54(1,27 ; 1,86)$ & $<0,001$ \\
\hline $40-49$ & 438 & 35,42 & $1,34(1,10 ; 1,62)$ & 0,003 & $1,46(1,18 ; 1,82)$ & 0,001 \\
\hline \multicolumn{7}{|l|}{ Estado conjugal } \\
\hline Casado ou união estável & 1.041 & 31,70 & 1,00 & & 1,00 & \\
\hline Solteiro $* \star \star$ & 502 & 28,09 & $0,89(0,75 ; 1,04)$ & 0,145 & $0,94(0,78 ; 1,12)$ & 0,467 \\
\hline 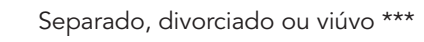 & 174 & 31,61 & $1,00(0,76 ; 1,28)$ & 0,982 & $1,20(0,93 ; 1,55)$ & 0,161 \\
\hline \multicolumn{7}{|l|}{ Anos de estudo formal } \\
\hline A partir de 12 & 739 & 35,45 & 1,00 & & 1,00 & \\
\hline $9-11$ & 568 & 30,63 & $0,86(0,72 ; 1,04)$ & 0,117 & $0,89(0,73 ; 1,08)$ & 0,232 \\
\hline $5-8$ & 252 & 24,21 & $0,69(0,53 ; 0,90)$ & 0,008 & $0,70(0,53 ; 0,90)$ & 0,008 \\
\hline Abaixo de 4 & 157 & 18,47 & $0,52(0,39 ; 0,69)$ & $<0,001$ & $0,51(0,36 ; 0,71)$ & $<0,001$ \\
\hline \multicolumn{7}{|l|}{ Renda familiar per capita } \\
\hline Acima de $\mathrm{R} \$ 1.500,00$ & 521 & 38,00 & 1,00 & & 1,00 & \\
\hline $\mathrm{R} \$ 510,00-\mathrm{R} \$ 1.499,00$ & 654 & 31,35 & $0,82(0,71 ; 0,96)$ & 0,015 & $0,92(0,77 ; 1,10)$ & 0,347 \\
\hline Abaixo de $\mathrm{R} \$ 510,00$ & 509 & 22,99 & $0,60(0,50 ; 0,74)$ & $<0,001$ & $0,81(0,64 ; 1,02)$ & 0,075 \\
\hline Boa ou muito boa & 1.370 & 32,19 & 1,00 & & 1,00 & \\
\hline Regular, ruim ou muito ruim & 347 & 24,50 & $0,76(0,63 ; 0,92)$ & 0,005 & $0,92(0,75 ; 1,13)$ & 0,413 \\
\hline \multicolumn{7}{|l|}{ Outro morador doador de sangue } \\
\hline Não & 1.122 & 28,25 & 1,00 & & 1,00 & \\
\hline Sim & 561 & 35,47 & $1,25(1,04 ; 1,51)$ & 0,018 & $1,35(1,13 ; 1,63)$ & 0,002 \\
\hline
\end{tabular}

IC95\%: intervalo de 95\% de confiança; RP: razão de prevalência, RP de 1 indica a categoria de referência.

* Regressão de Poisson;

** RP de 1,00 indica a categoria de referência;

$\star \star \star$ Poder da amostra $<80 \%$.

3\% da população a cada ano. Em países da Ásia e África, todavia, essa taxa se situa abaixo de $2 \% 1$. A taxa de doações de sangue em países da América do Sul com Índice de Desenvolvimento Humano semelhante ao Brasil, como Peru, Colômbia e Equador, não ultrapassa $2 \% 1$. No Brasil, ela varia de $1,5 \%$ (regiões Norte e Nordeste) a 2,6\% (Região Centro-oeste), sendo a média nacional $1,9 \%$ ao ano 3 ; porém períodos de férias, frio ou chuvas podem diminuir as doações em até $25 \% 4$. A OMS estima que uma taxa de doação entre $3 \%$ e $5 \%$ é suficiente para suprir as necessidades de uma área urbana 17. A prevalência de doação nos últimos 12 meses encontrada na população adulta da área urbana de Florianópolis esteve acima desse nível e do observado em outras regiões do país. No entanto, dados não publicados do Hemocentro de Santa Catarina, com base no volume de doações captado, sugerem que a taxa de doação na grande Florianópolis não ultrapasse 1,5\% ao ano. Embora seja possível que os moradores da área urbana de Florianópolis doem sangue em outros hemocentros, ou que a prevalência de doação de sangue naquela área 
Fatores associados à doação de sangue nos últimos 12 meses. Município de Florianópolis, Santa Catarina, Brasil, 2009.

\begin{tabular}{|c|c|c|c|c|c|c|}
\hline \multirow[t]{2}{*}{ Variável } & \multirow[t]{2}{*}{$\mathrm{n}$} & \multirow[t]{2}{*}{ Prevalência (\%) } & \multicolumn{2}{|c|}{ Análise bruta * } & \multicolumn{2}{|c|}{ Análise ajustada * } \\
\hline & & & $\mathrm{RP} * \star(I C 95 \%)$ & Valor de $p$ & $\mathrm{RP} * \star(I C 95 \%)$ & Valor de $p$ \\
\hline \multicolumn{7}{|l|}{ Sexo } \\
\hline Masculino & 760 & 7,89 & 1,00 & & 1,00 & \\
\hline Feminino & 957 & 4,91 & $0,62(0,39 ; 0,99)$ & 0,045 & $0,63(0,40 ; 1,01)$ & 0,055 \\
\hline \multicolumn{7}{|l|}{ Cor da pele autorreferida } \\
\hline Branca & 1.441 & 5,90 & 1,00 & & 1,00 & \\
\hline 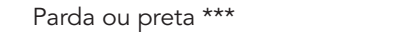 & 234 & 8,55 & $1,45(0,88 ; 2,37)$ & 0,138 & $1,55(0,94 ; 2,55)$ & 0,086 \\
\hline \multicolumn{7}{|l|}{ Idade (anos) } \\
\hline $20-29$ & 540 & 10,74 & 1,00 & & 1,00 & \\
\hline $30-39$ & 391 & 5,88 & $0,55(0,33 ; 0,91)$ & 0,021 & $0,76(0,41 ; 1,43)$ & 0,394 \\
\hline $40-49$ & 438 & 4,34 & $0,40(0,25 ; 0,65)$ & $<0,001$ & $0,66(0,36 ; 1,22)$ & 0,183 \\
\hline $50-59$ & 348 & 2,01 & $0,18(0,09 ; 0,40)$ & $<0,001$ & $0,32(0,31 ; 0,77)$ & 0,012 \\
\hline \multicolumn{7}{|l|}{ Estado conjugal } \\
\hline Casado ou união estável & 1.041 & 4,32 & 1,00 & & 1,00 & \\
\hline Solteiro & 502 & 11,35 & $2,63(1,78 ; 3,88)$ & $<0,001$ & $1,66(1,02 ; 2,69)$ & 0,041 \\
\hline Separado, divorciado ou viúvo *** & 174 & 2,87 & $0,66(0,30 ; 1,48)$ & 0,314 & $0,02(0,44 ; 2,34)$ & 0,961 \\
\hline \multicolumn{7}{|l|}{ Anos de estudo formal } \\
\hline A partir de 12 & 739 & 8,93 & 1,00 & & 1,00 & \\
\hline $9-11$ & 568 & 6,16 & $0,69(0,44 ; 1,08)$ & 0,098 & $0,67(0,42 ; 1,07)$ & 0,090 \\
\hline $5-8$ & 252 & 2,38 & $0,27(0,11 ; 0,66)$ & 0,005 & $0,34(0,12 ; 0,97)$ & 0,045 \\
\hline Abaixo de 4 & 157 & 0,00 & $<0,01(<0,01 ;<0,01)$ & $<0,001$ & $<0,01(<0,01 ;<0,01)$ & $<0,001$ \\
\hline \multicolumn{7}{|l|}{ Renda familiar per capita } \\
\hline Acima de $\mathrm{R} \$ 1.500,00$ & 521 & 7,49 & 1,00 & & 1,00 & \\
\hline $\mathrm{R} \$ 510,00-\mathrm{R} \$ 1.499,00 * \star \star$ & 654 & 6,42 & $0,86(0,55 ; 1,33)$ & 0,450 & $0,95(0,59 ; 1,52)$ & 0,822 \\
\hline Abaixo de $\mathrm{R} \$ 510,00$ & 509 & 4,91 & $0,66(0,38 ; 1,13)$ & 0,128 & $0,94(0,49 ; 1,83)$ & 0,866 \\
\hline Regular, ruim ou muito ruim & 347 & 3,46 & $0,50(0,30 ; 0,82)$ & 0,007 & $0,82(0,47 ; 1,41)$ & 0,459 \\
\hline \multicolumn{7}{|l|}{ Outro morador doador de sangue } \\
\hline Não & 1.122 & 5,53 & 1,00 & & 1,00 & \\
\hline Sim & 561 & 7,84 & $1,42(1,00 ; 2,04)$ & 0,057 & $1,35(0,93 ; 1,96)$ & 0,117 \\
\hline
\end{tabular}

IC95\%: intervalo de 95\% de confiança; RP: razão de prevalência.

* Regressão de Poisson;

** RP de 1,00 indica a categoria de referência;

*** Poder da amostra $<80 \%$.

seja diferente daquela na região metropolitana, deve ser lembrado que os resultados obtidos dependeram da confiabilidade dos participantes. Vieses como prevaricação, em que o sujeito refere comportamentos falsos, porém aceitáveis socialmente, e telescópico, em que ele acredita serem mais recentes fatos relevantes distantes no tempo, como a última doação de sangue, podem aumentar a prevalência estimada, distante do parâmetro real.

Tornar a doação um comportamento habitual é um dos objetivos do incentivo à doação de sangue 1. A doação de repetição apresenta menor risco para transmissão de doenças infecciosas 18,19 , além de menor recusa na triagem clínica 20,21 . Isso ocorre porque, já tendo sido aprovado anteriormente, a chance de recusa é menor do que na primeira doação. Não obstante, a doação de repetição pode responder por importante parcela do total de sangue doado. Em estudo com população americana, foi observado que $80 \%$ do suprimento de sangue haviam sido obtidos de doações de repetição 22 . Em Pelotas, $47 \%$ dos participantes que referiram haver doado sangue no 
Participantes que referiram doação de sangue nos últimos 12 meses, distribuição por faixa etária. Município de Florianópolis, Santa Catarina, Brasil, 2009

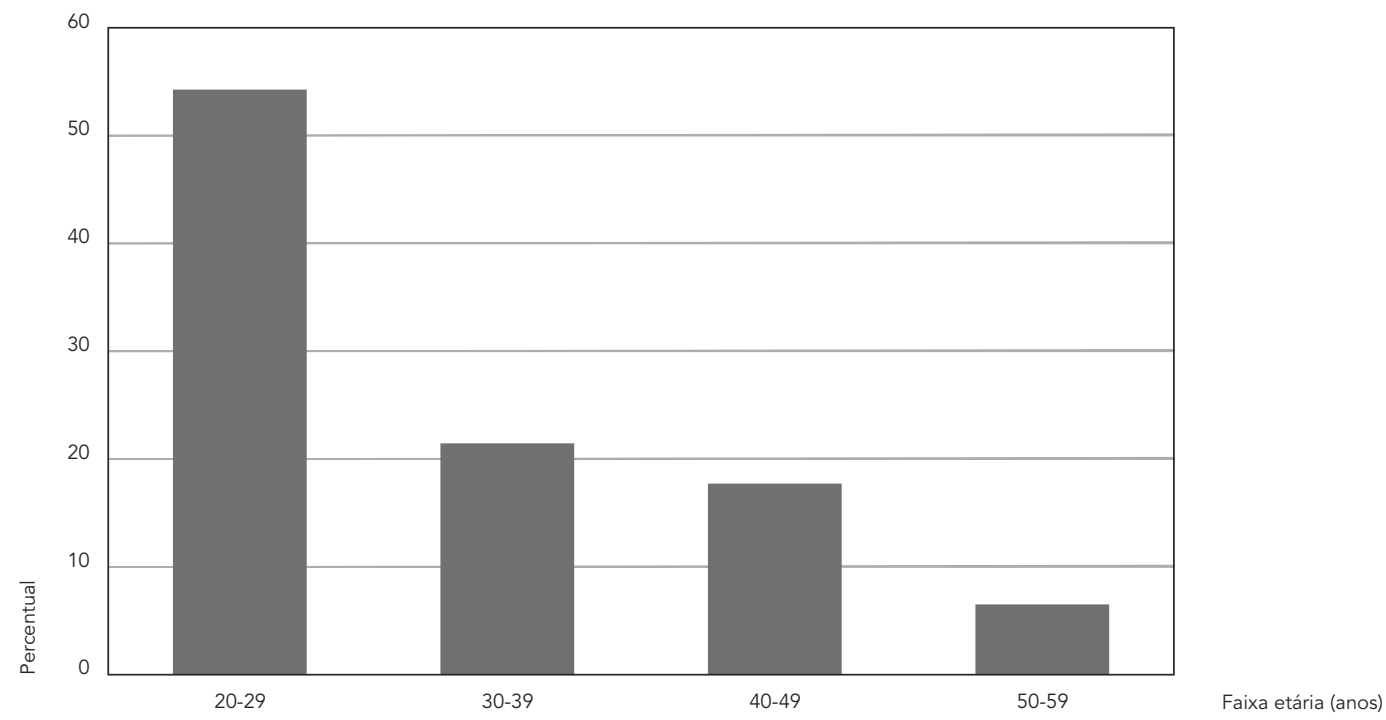

último ano também referiram haver doado mais de uma vez ao ano 7 . Na nossa casuística, a proporção de doação de repetição foi semelhante àquela descrita em Pelotas, entretanto acreditamos ela possa ser aumentada, mediante programas de recrutamento específicos.

Outro desafio é aumentar o número de doações espontâneas. Dados consistentes apontam que elas também são mais seguras para evitar transmissão de doenças infecciosas do que doações remuneradas, ou mesmo aquelas para reposição 1,20. Entretanto, estudo realizado em três grandes centros de coleta no Brasil acrescentou mais um nível de complexidade. Em São Paulo, Minas Gerais e Pernambuco, entre 2007 e 2008, foi demonstrado que primodoadores com motivação para reposição eram mais seguros quanto ao risco para transmissão de HIV do que primodoadores com motivação espontânea. Além disto, naquele estudo, a prevalência de infecção pelo HIV não esteve associada com a motivação para doação (espontânea ou reposição) entre doadores de repetição ${ }^{5}$. Estimativas em centros de coleta de diversas regiões do país sugerem que a contribuição das doações para reposição varia entre $19 \%$ a $52 \%$ 3,23. Em nosso estudo, a proporção de participantes que referiram doação para reposição foi menor do que naqueles centros. Diferenças culturais, maior escolaridade e faixa de renda podem ter influenciado o resultado. Ainda que nenhum participante que referiu doação nos últimos 12 meses tenha manifestado doação pela expectativa de receber resultados de exames sobre seu estado de saúde, limitações relacionadas ao poder da amostra e ao viés de prevaricação não permitem análise adequada desse resultado.

Em Florianópolis, a distribuição por faixa etária dos doadores que manifestaram doação nos últimos 12 meses foi diferente do restante do país, onde é estimado que a maioria dos doadores de sangue apresentem idade superior a 29 anos $3,7,23$. Os motivos pelos quais os doadores em Florianópolis são mais jovens não estão claros. De qualquer modo, tal associação, em longo prazo, pode gerar diminuição dos estoques de sangue 24,25 . Isso ocorre porque com o envelhecimento dos indivíduos diminuem os doadores e aumenta a demanda por procedimentos médicos e sangue.

Nossos resultados reforçam a impressão de que os doadores de sangue possuem características à parte da população geral. O conhecimento dessas características pode auxiliar no planejamento de ações voltadas à captação de doadores e diminuição do risco para transmissão de doen- 
ças infecciosas pelo sangue. Em Florianópolis, nosso estudo indica a necessidade de esforços para a manutenção da atividade de doação, es- tímulo à doação espontânea e de repetição, e recrutamento de doadores com maior faixa etária.

\section{Resumen}

Los principales objetivos fueron estimar la prevalencia de la donación de sangre e identificar los factores asociados. Se realizó un estudio transversal con base poblacional ( $n=1.720$ ), incluyendo personas de edades entre 20 y 59 años, que viven en Florianópolis, sur de Brasil. Los participantes informaron que donaron alguna vez en el 30,6\% de los casos, y en los últimos 12 meses en el 6,2\% de los casos. De ellos, un 31,8\% informó de repetición en la donación, el 80,4\% informó al menos una donación voluntaria, y el 15,9\% al menos una donación de reposición. De acuerdo con la regresión de Poisson ( $p<0,05)$, el sexo masculino, el color de la piel negro o pardo, mayor edad, mayor nivel educativo, y la habitación compartida con otro donante se asociaron con la donación alguna vez. Una menor edad y el estado civil solo se asociaron con al menos una donación en los últimos 12 meses. Constatamos una mayor prevalencia de la donación de sangre en el último año y un mayor porcentaje de donantes jóvenes de este grupo, así como un menor porcentaje de donantes de reposición, respecto a las cifras publicadas en Brasil.

Donantes de Sangre; Adulto; Prevalencia; Estudios Transversales

\section{Colaboradores}

R. M. G. Silva, E. Kupek e K. G. Peres contribuíram na concepção e projeto, análise e interpretação dos dados, redação do artigo e revisão crítica relevante ao conteúdo, e aprovação final da versão a ser publicada.

\section{Agradecimentos}

Agradecemos aos técnicos do Instituto Brasileiro de Geografia e Estatística e da Secretaria Municipal de Saúde de Florianópolis pelo auxílio na operacionalização deste estudo. 


\section{Referências}

1. World Health Organization. Blood safety and availability. http://www.who.int/mediacentre/ factsheets/fs279/en/index.htm (acessado em 15/ Jun/2012).

2. Sullivan MT, Cotten R, Read EJ, Wallace EL. Blood collection and transfusionin the United States in 2001. Transfusion 2007; 47:385-94.

3. Coordenação Geral de Sangue e Hemoderivados, Secretaria de Atenção à Saúde, Ministério da Saúde. Sangue e hemoderivados produção hemoterápica. Brasília: Ministério da Saúde; 2011.

4. Ministério da Saúde. Ministério alerta para redução no estoque de sangue. http://portal.saude.gov. $\mathrm{br} /$ aplicacoes/noticias/default.cfm? (acessado em 15/Nov/2012).

5. Sabino EC, Gonçalez TT, Carneiro-Proietti AB, Sarr M, Ferreira JE, Sampaio DA, et al. Human immunodeficiency virus prevalence, incidence, and residual risk of transmission by transfusions at Retrovirus Epidemiology Donor Study-II blood centers in Brazil. Transfusion 2012; 52:870-9.

6. Kupek E, Petry A. Comparison of epidemiological methods for estimation of hepatitis B incidence and residual risk for blood donors in southern Brazil. Journal of Transfusion 2011. http://www.hindawi. com/journals/jbt/2011/985383/cta/ (acessado em 15/Nov/2012).

7. Zago A, da Silveira MF, Dumith SC. Blood donation prevalence and associated factors in Pelotas, Southern Brazil. Rev Saúde Pública 2010; 44: $112-20$.

8. Landis JR, Koch GG. The measurement of observer agreement for categorical data. Biometrics 1977; 33:159-74.

9. Javadzadeh Shahshahani H, Yavari MT, Attar M, Ahmadiyeh MH. Knowledge, attitude and practice study about blood donation in the urban population of Yazd, Iran, 2004. Transfus Med 2006; 16:403-9.

10. Zaller N, Nelson KE, Ness P, Wen G, Bai X, Shan H. Knowledge, attitude and practice survey regarding blood donation in a Northwestern Chinese city. Transfus Med 2005; 15:277-86.

11. Gillespie TW, Hillyer CD. Blood donors and factors impacting the blood donation decision.Transfus Med Rev 2002; 16:115-30.

12. Misje AH, Bosnes V, Gåsdal O, Heier HE. Motivation, recruitment and retention of voluntary nonremunerated blood donors: a survey-based questionnaire study. Vox Sang 2005; 89:236-44.

13. Wu Y, Glynn SA, Schreiber GB, Wright DJ, Lo A, Murphy EL, et al. First-time blood donors: demographic trends. Transfusion 2001; 41:360-4.
14. James AB, Hillyer CD, Shaz BH. Demographic differences in estimated blood donor eligibility prevalence in the United States. Transfusion 2012; 52:1050-61.

15. Shaz BH, Hillyer CD. Minority donation in the United States: challenges and needs. Curr Opin Hematol 2010; 17:544-9.

16. Ajzen I, Madden TJ. Prediction of goal-directed behavior: attitudes, intentions, and perceived behavioral control. J Exp Soc Psychol 1986; 22:453-74.

17. Ministério da Saúde. Saúde incentiva doações de sangue. http://portal.saude.gov.br/portal/saude/ visualizar_texto.cfm?idxt=25472 (acessado em 20/ Nov/2012).

18. Sullivan P. Developing an administrative plan for transfusion medicine: a global perspective. Transfusion 2005; 45(4 Suppl):224S-40.

19. Dodd RY, Notari 4th EP, Stramer SL. Current prevalence and incidence of infectious disease markers and estimated window-period risk in the American Red Cross blood donor population. Transfusion 2002; 42:975-9.

20. Newman B. Blood donor suitability and allogeneic whole blood donation. Transfus Med Rev 2001; 15:234-44.

21. Zou S, Eder AF, Musavi F, Notarilv EP, Fang CT, Dodd RY, et al. Implementation of the Uniform Donor History Questionnaire across the American Red Cross Blood Services: increased deferral among repeat presenters but no measurable impact on blood safety. Transfusion 2007; 47:1990-8.

22. Zou S, Musavi F, Notari 4th EP, Fang CT; ARCNET Research Group. Changing age distribution of the blood donor population in the United States. Transfusion 2008; 48:251-7.

23. Carneiro-Proietti AB, Sabino EC, Sampaio D, Proietti FA, Gonçalez TT, Oliveira CD, et al. Demographic profile of blood donors at three major Brazilian blood centers: results from the International REDS-II study, 2007 to 2008. Transfusion 2010; 50:918-25.

24. Rodrigues RN. Editorial. Rev Bras Estud Popul 2010; $27: 247-9$.

25. Greinacher A, Fendrich K, Brzenska R, Kiefel V, Hoffmann W. Implications of demographics on future blood supply: a population-based crosssectional study. Transfusion 2011; 51:702-9.

Recebido em 05/Dez/2012

Versão final reapresentada em 01/Abr/2013 Aprovado em 25/Abr/2013 